\title{
Recurrent aortic-arch pseudoaneurysm eroding into the left main bronchus following descending thoracic aortic stent repair
}

\section{Lijun Duan}

The First Hospital of Jilin University

\section{Ruonan Pan}

The First Hospital of Jilin University

Xiaoqian Lu

The First Hospital of Jilin University

Dianbo Cao ( $\square$ caodb@jlu.edu.cn )

The First Hospital of Jilin University

\section{Case Report}

Keywords: Behcet's disease, Vascular complications, Endovascular techniques, CT angiography

Posted Date: March 2nd, 2022

DOI: https://doi.org/10.21203/rs.3.rs-1398916/v1

License: (c) (i) This work is licensed under a Creative Commons Attribution 4.0 International License. Read Full License 


\section{Abstract}

Background: Behcet's disease (BD) is a form of systematic vasculitis that affects vessels of various sizes anywhere in the body. Aortic pseudoaneurysm is the most hazardous lesion in Behcet's disease (BD) and is associated with high mortality rates once rupture.

Case presentation: In this report, we present a patient with known history of Behcet's disease (BD), whose recurrent aortic-arch pseudoaneurysm eroding into the left main bronchus was identified after a 4-year duration of endovascular aortic repair (EVAR) for thoracic descending aortic pseudoaneurysm ruptured into the left lung. Repeated endovascular aortic repair (EVAR) combined with the chimney stent effectively controlled massive haemoptysis under life-threatening conditions, and the patient was good condition at the 5-year follow-up. Conclusions: Endovascular aortic repair (EVAR) can be used as an effective and problem-solving treatment approach for thoracic aortic aneurysms (TAA) eroding into the lung, even recurrent pseudoaneurysm after endovascular aortic repair (EVAR) in BD patients. Among the imaging techniques assessing the technical success, outcome and complications, CT angiography (CTA) offers a fast, accessible and sensitive imaging modality.

\section{Background}

Behcet's disease(BD), while originally described as a triad of oral and genital ulcerations and relapsing ocular inflammation, has more to it than just that[1]. BD could affect various types of vessels manifesting as venous thrombosis and arterial pseudoaneurysm formation. Aortic involvement is one of the most severe manifestations in $\mathrm{BD}$, and aortic aneurysm rupture has become the principal cause of death in $\mathrm{BD}$ with vascular involvement[2, 3]. The abdominal aorta is the most common site of pseudoaneurysm formation, while thoracic aorta is a relative rare location. Aortic pseudoaneurysm may be found as the first symptom, or comes after previous treatment. Massive life-threatening hemoptysis occasionally occurs only when thoracic aortic pseudoaneurysm ruptures into the lung parenchyma or tracheobronchial trees. Traditional open repair of aortic aneurysm in BD patients is associated with a high recurrence rate of pseudoaneurysms at anastomotic sites[4]. Endovascular aneurysm repair(EVAR) provides a favorable alternative for BD patients, as it refrains from the need for surgical aortic anastomosis. Even so, recurrent pseudoaneurysm is still a serious problem in BD patients[5]. Among the imaging techniques assessing the technical success, outcome and complications, noninvasive CT angiography(CTA) offers a fast, accessible and sensitive imaging modality. We herein report a case of a patient with BD who developed recurrent pseudoaneurysm eroding into the left main bronchus after EVAR for thoracic descending aortic pseudoaneurysm ruptured into the left lung. Repeated EVAR combined with the chimney stent provided an effective strategy in controlling massive hemoptysis from aortic-arch pseudoaneurysms under lifethreatening conditions.

\section{Case Presentation}


A 47-year-old man with a confirmed 10-year diagnosis of BD was admitted to our hospital due to hoarse voice, recurrent hemoptysis and dyspnea on exercise for about 2 weeks. The patient was diagnosed to have a thoracic aortic saccular aneurysm ruptured into the left lung on CTA 4 years previously(Fig-1,2) and was be treated by EVAR. He recovered uneventfully and hemoptysis disappeared. Approximately 12 months after EVAR, follow-up CTA indicated that stent was well positioned and poorly attached to the concave aspect of aortic arch at the proximal part(Fig-3), predicting the possibility of future aneurysm. On physical examination, his blood pressure was $122 / 69 \mathrm{mmHg}$, and heart rate was 100 beats per minute. Decreased breath sound were heard in the left lower-middle lung zone. The hemoglobin level was $109 \mathrm{~g} / \mathrm{L}$, C-reactive protein was $2.0 \mathrm{mg} / \mathrm{L}$, and erythrocyte sedimentation rate was $10 \mathrm{~mL} /$ hour. Chest CTA scan showed partial left lung atelectasis and serious stenosis of the left main brochus invaded by aortic-arch pseudoaneurysm(Fig-4,5) with significant increase in size compared with previous CT image. After multidisciplinary consultation, an emergent endovascular repair was performed. Under general anesthesia, the patient's right femoral artery was dissected and controlled. An endovascular stent graft (160mmX36mm; XianJian, China) was anchored to the ascending side of aortic arch, thus covering the site of poor attachment of previous stent and the orifice of the left common carotid artery and left subclavian artery(Fig-6,7). Then, self-expanding stent $(60 \mathrm{mmx} 10 \mathrm{~mm}$; Gore viabahn, America) with chimney technique was retrogradely placed at the ostium of the left common carotid artery to preserve its perfusion through transcarotid approach. Repeat angiography showed complete occlusion of the aneurysm and the procedure was terminated. On the tenth day of postoperative management, carotid color doppler showed left subclavian steal syndrome from retrograde blood flow of the left vertebral artery. No change in the patient's neurologic status was present during the perioperative period and he was discharged 14 days later in a stable condition. Lacking the disease activity, he did not take any immunosuppressive medication. Up to now, he has been followed for 60 months with a favorable chest CTA outcome(Fig-8), and no any complications have been detected.

\section{Discussion And Conclusions}

Behcet's disease(BD) is a rare, chronic, autoimmune, multisystem disorder that can cause inflammation of blood vessels anywhere in the body. Studies are still being conducted in attempts to disclose the pathogenesis of this disease, which remains unclear so far. BD could affect various types of vessels and cause variable kinds of pathologies. As arterial complications, development of true or false aneurysms in great vessels accounts for $1-7 \%$ of $B D$ patients[6]. The common sites of aneurysm related to BD have been reported as abdominal aortic aneurysm, pulmonary aneurysm, carotid artery aneurysm, and lower extremity aneurysm. However, aortic pseudoaneurysm is the most catastrophic lesion in BD, and aortic aneurysm rupture has become the principal cause of death in $\mathrm{BD}$ with vascular complications.

Aneurysm formation of thoracic aorta is a rare but serious manifestation of BD. If thoracic aortic aneurysms(TAA) is ruptured into the lung parenchyma or tracheobronchial tree, patients would suffer massive life-threatening hemoptysis, different from those common causes such as bronchiectasis, tuberculosis, fungal infections, and cancer. There was not any other evidence except for a definite history of BD in our patient when TAA was firstly identified to be responsible for initial hemoptysis, which 
suggested that the vascular lesion was not an isolated event but was related to BD. Practically, detailed medical history and careful physical examination is of great value in occult onset patients. More interestingly, recurrent hemoptysis occurred 4 years later due to aortic-arch pseudoaneurysm erosion into the left main bronchus based on CT evaluation. According to our speculation, fistula developed between aorta and tracheobronchial tree possibly because erosion from the continuous pulsatile pressure from blood flow and progressive enlargement of pseudoaneurysm resulted in necrosis and compression of the main bronchus. Radiographic surveillance like CT angiography provides the direct evidence of the two episodes of hemoptysis in this patient.

The major problem of open surgical treatment for BD-associated aortic pseudoaneurysms is its tendency to develop recurrent false aneurysms at the anastomosis sites, which occur in 30 to $50 \%$ of patients[3]. EVAR, introduced in the mid-1990s, provides a less invasive approach in treating acute and chronic thoracic aortic pathology with reduced surgery time and a more rapid recovery. Additionally, It also causes less blood loss and has lower mortality rates. For these reasons, recent studies strongly favor endovascular repair over conventional open surgical repair in conjunction with administration of systemic immunosuppressive and antiinflammatory therapy before and after the procedure in the active phase of $B D[7-9]$. Even so, recurrent pseudoaneurysm is still a serious problem in BD patients[10]. In our study, the patient's lesion morphology was suitable for endovascular repair based on the adequate anchor zones at his initial detection. His recurrent aortic-arch pseudoaneurysm was proposed to be associated with poor attachment of the proximal stent based on the appearance of 1-year follow-up CTA. Meanwhile, vessel wall injuries or mechanical stress at the edge of the stent graft may trigger tissue inflammation with arterial pulsation. Recurrent pseudoaneurysms at the margin of the stent graft require timely intervention with additional stent graft components.

With continuous improvements of stent grafts and technical experiences, indications of EVAR for aortic pathology are constantly enlarged. For those vascular lesions involving the aortic arch, important arterial branches, and a short anchor zone, use of a common configuration of stent graft often leads to various types of severe complications. Parallel to the main aortic stent graft, the chimney technique uses a covered or bare stent to maintain blood flow to the vital organs, which could be used to treat lesions with inadequate anchor zones. In our patient, the chimney stent was implanted into the left common carotid artery, while the left subclavian artery was sacrificed. A reversal of the left vertebral flow to left subclavian artery was observed by postoperative carotid doppler, indicating the occlusion of its proximal end. EVAR combination with the chimney stent is a valid solution to the critical location of aortic aneurysm in emergency setting. A study combining EVAR with the chimney technique revealed that the chimney-graft technique for aortic-arch pathologies is technically applicable in both elective and emergency situations, and is associated with satisfactory perioperative outcomes with a success rate of $90.2 \%[11]$. Our successful management suggests that this approach may be an attractive therapeutic alternative to treat aortic-arch pseudoaneurysm for those high-risk surgical candidates. Meanwhile, the placement of endovascular stent should be such that the proximal end does not lie in the curve of the proximal descending thoracic aorta, and it should be either positioned more proximally into the arch or distally into 
the straight portion of the thoracic aorta, as the lesson learned from our patient. The precise evaluation for therapeutic effect is easily obtained by CTA[12], similar to our case.

In conclusion, TAA related to BD is rarely encountered, which is one of the rarest causes of life-threatening hemoptysis. EVAR can be used as an effective and problem-solving treatment approach for TAA eroding into the lung, even recurrent pseudoaneurysm after EVAR in BD patients. EVAR combined with the chimney technique widens the indication of EVAR for aortic pathology. Among the imaging techniques assessing the technical success, outcome and complications, CTA offers a fast, accessible and sensitive imaging modality.

\section{Declarations}

\section{Ethics approval and consent to participate}

All methods were performed in accordance with the Declaration of Helsinki. The study was approved by the Medical Ethics Committee of the First Hospital of Jilin University.

\section{Consent for publication}

The study was informed written consent was obtained from the patient for publication of this case report and accompanying images.

\section{Availability of data and materials}

Data sharing is not applicable to this article as no datasets were generated or analysed during the current study.

\section{Competing interests}

All authors disclosed no conflicts.

\section{Funding}

Self-paying

\section{Authors' contributions}

RNP looked up and analyzed theories of behcet's disease. LJD explained the special aspects of the case and was a major contributor in writing the manuscript. All authors read and approved the final manuscript.

\section{Acknowledgements}

Not applicable. 


\section{References}

1. Fok M, Bashir M, Goodson N, et al. Thoracic aortic aneurysms in Behçet's disease. Rheumatology (Oxford). 2017;56(4):501-502.

2. Marone EM, Diaco DA, Brioschi C, et al. Emergent Endovascular Treatment of Thoracoabdominal Aortic Rupture in Behcet's Disease. Ann Vasc Surg. 2018;47:282.e7-e11.

3. Kural-Seyahi E, Fresko I, Seyahi N, et al. The long-term mortality and morbidity of Behçet syndrome: a 2-decade outcome survey of 387 patients followed at a dedicated center. Medicine (Baltimore) 2003;82:60-76.

4. Hosaka A, Miyata $T$, Hoshina $\mathrm{K}$, et al. Prognosis of arterial aneurysm after surgery in patients with Behçet's disease. Int Angiol. 2014;33(5):419-425.

5. Honda S, Hirano F, Mouri M, et al. Aneurysm formation after stent grafting in vascular Behçet's disease. Arthritis Rheumatol. 2018;70(2):322.

6. Gürler A, Boyvat A, Türsen U. Clinical manifestations of Behçet's disease: an analysis of 2147 patients. Yonsei Med J. 1997;38(6):423-427.

7. Shen C, Li W, Zhang Y, et al. Outcomes of surgery for patients with Behcet's disease causing aortic pseudoaneurysm: a shift from open surgery to endovascular repair. Clinics (Sao Paulo). 2016;71(6):302-310.

8. Balcioglu O, Ertugay S, Bozkaya $\mathrm{H}$, et al. Endovascular Repair and Adjunctive Immunosuppressive Therapy of Aortic Involvement in Behçet's Disease. Eur J Vasc Endovasc Surg. 2015;50(5):593-598.

9. Park JH, Chung JW, Joh JH, et al. Aortic and arterial aneurysms in behçet's disease: management with stent-grafts-initial experience. Radiology. 2001;220(3):745-750.

10. Zhang SH, Zhang FX. Behcet's disease with recurrent thoracic aortic aneurysm combined with femoral artery aneurysm: a case report and literature review. J Cardiothorac Surg. 2017;12(1):79.

11. Yang J, Xiong J, Liu X, et al. Endovascular chimney technique of aortic arch pathologies: $A$ systematic review. Ann Vasc Surg. 2012;26(7):1014-1021.

12. Qi L, Cai J, Mao D, et al. Use of contrast-enhanced computed tomographic imaging to diagnose and evaluate Behçet's disease with vascular complications. Exp Ther Med. 2019;18(6):4265-4272.

\section{Figures}

\section{Figure 1}

Contrast-enhanced CT scan revealed a $21 \times 30 \mathrm{~mm}$ saccular aneurysm with rupture into the superior segment of left lung lower lobe. 
Figure 2

Contrast-enhanced CT scan revealed a $21 \times 30 \mathrm{~mm}$ saccular aneurysm with rupture into the superior segment of left lung lower lobe.

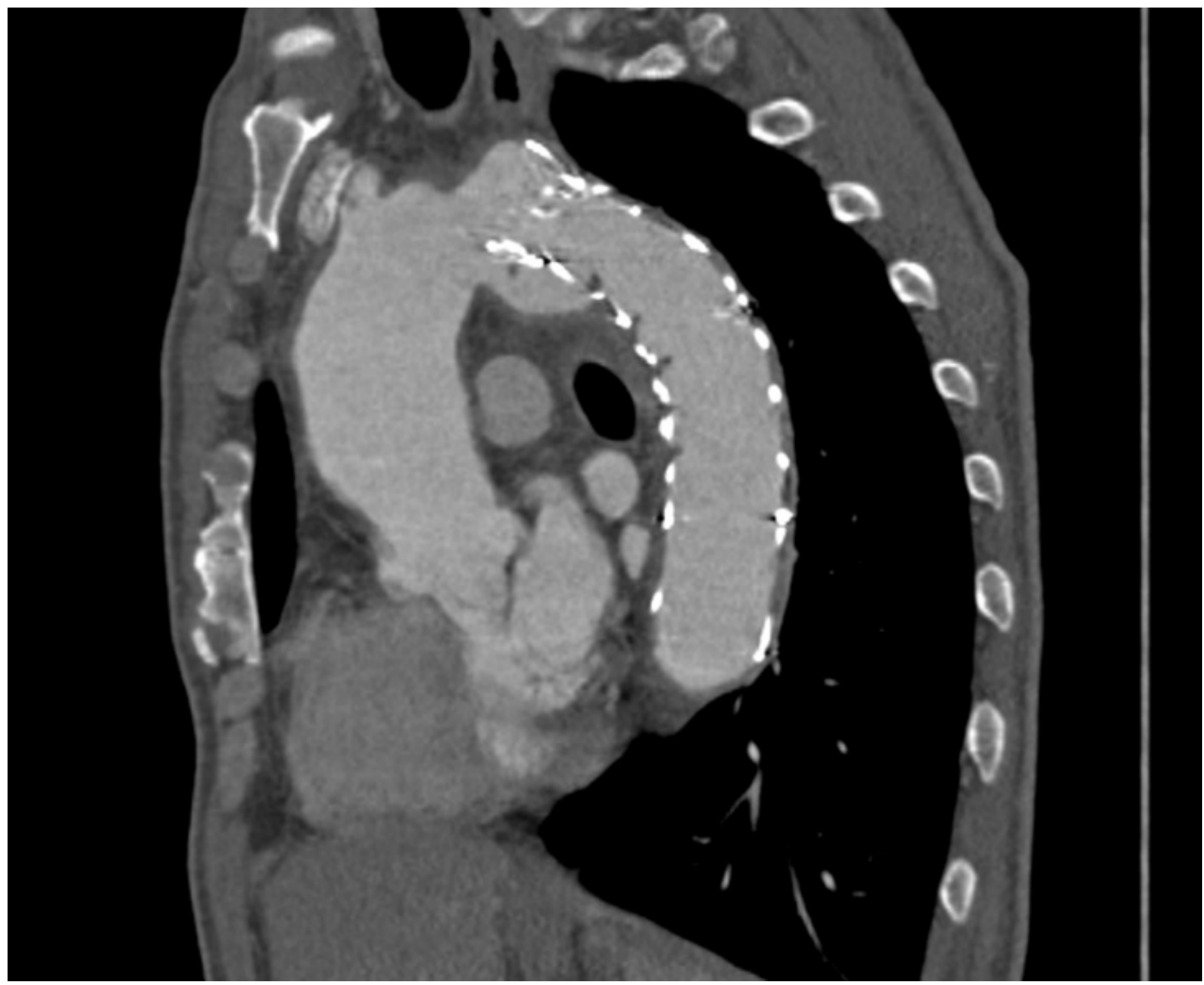

\section{Figure 3}

Follow-up CTA one year later demonstrated that the proximal stent was poorly attached to the concave side of aortic arch.

\section{Figure 4}

Chest CTA showed serious stenosis of the left main brochus and partial atelectasis of left lung due to aortic-arch pseudoaneurysm eroding into the main bronchus. 


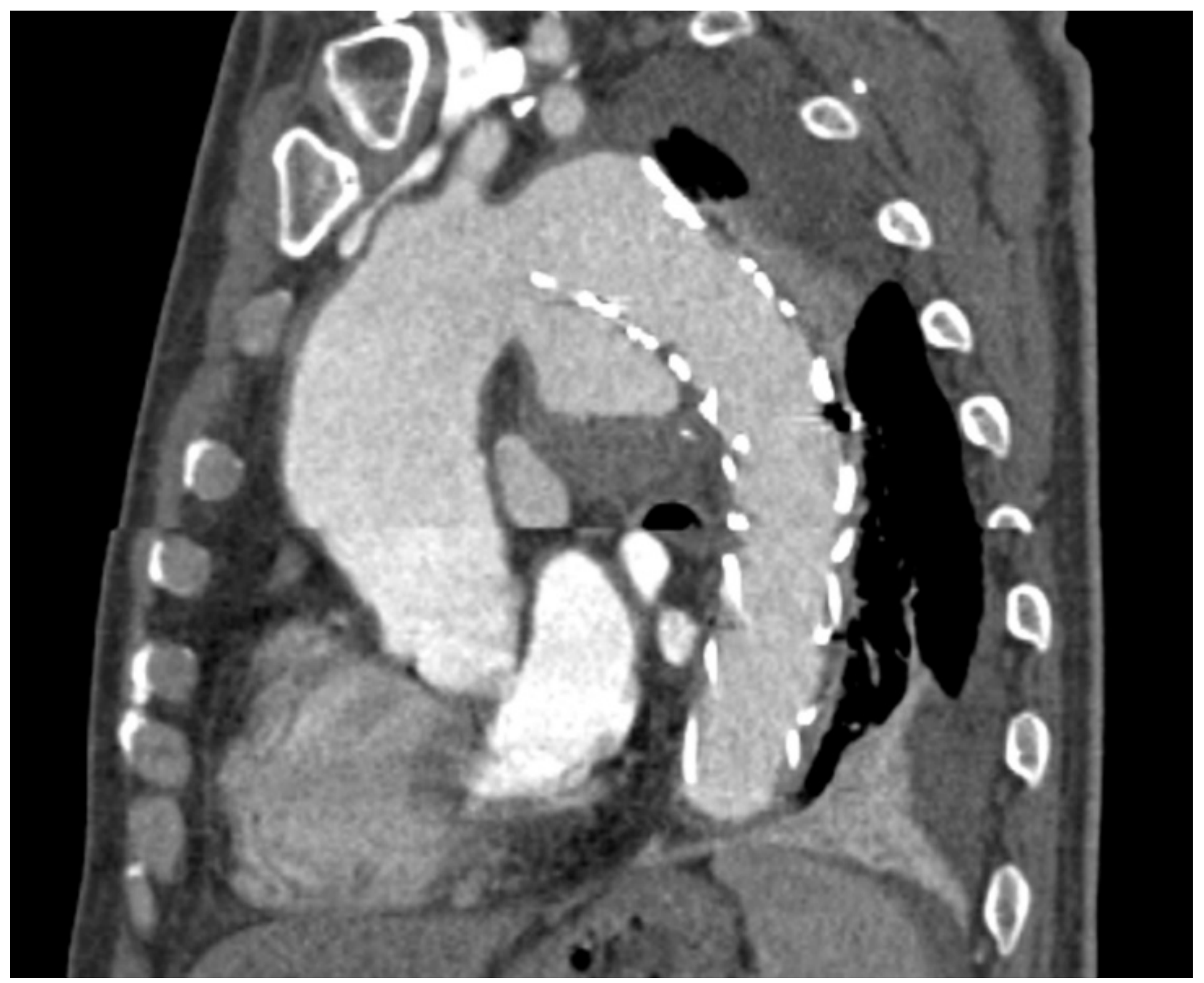

Figure 5

Chest CTA showed serious stenosis of the left main brochus and partial atelectasis of left lung due to aortic-arch pseudoaneurysm eroding into the main bronchus. 


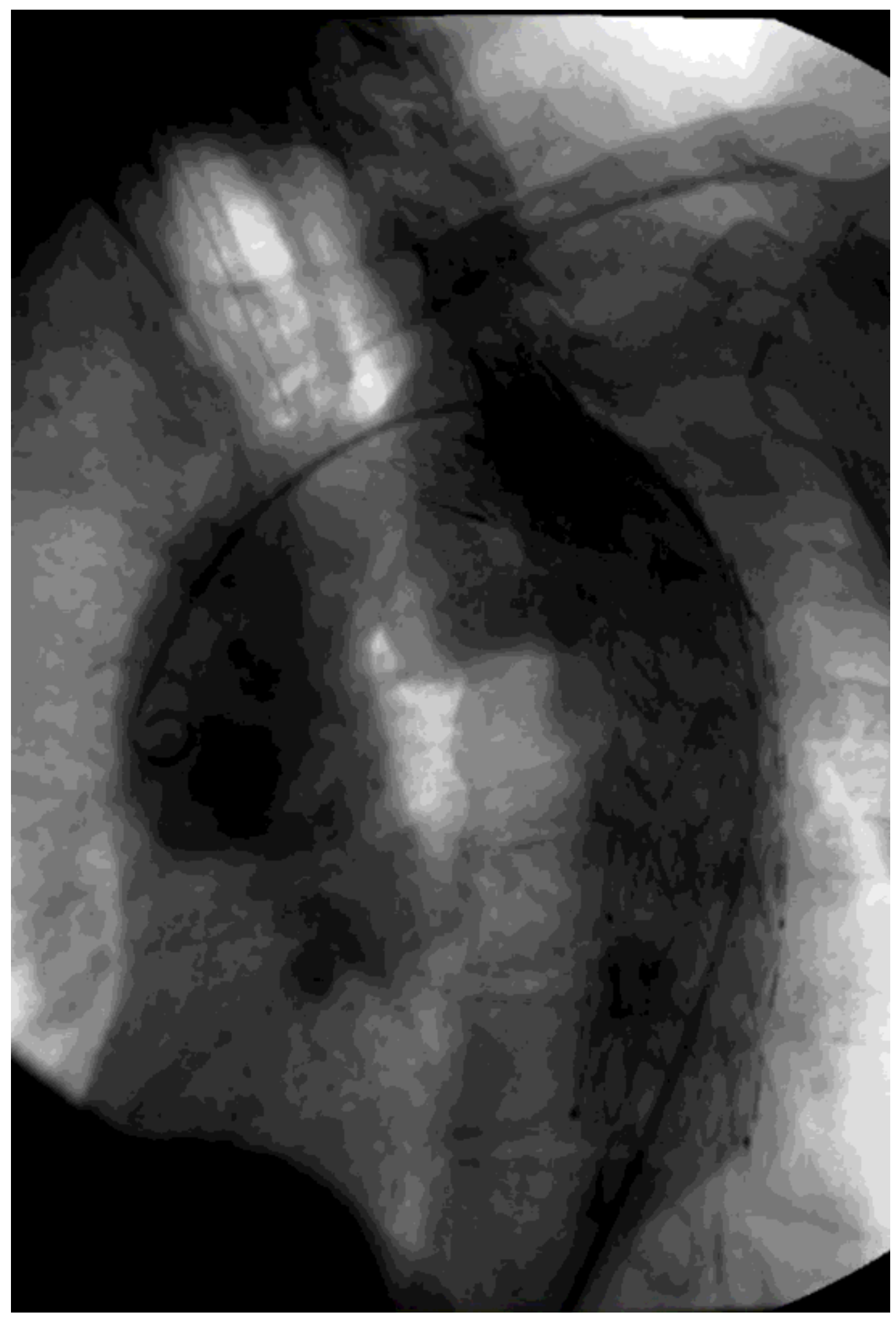

\section{Figure 6}

Intraoperative angiogram show saccular aneurysms located at the concave side of aortic arch and eroding into the left main bronchus.

Figure 7 
A completion angiogram showed complete occlusion of aortic-arch pseudoaneurysm.

\section{Figure 8}

Postoperative CTA confirmed complete regression of the aortic-arch pseudoaneurysm, no bronchial stenosis, and the patency of chimney stent. 